\title{
Throughput Enhancement for Better QoS in WCDMA Networks
}

\author{
Vandana Khare \\ Associate Professor ECE \\ CMR College of Engg and \\ Technology \\ Hyderabad, India
}

\author{
Y. Madhavee Latha, Ph.D. \\ Professors and Principal \\ MRECW \\ Secunderabad, India
}

\author{
D. Srinivasa Rao, Ph.D. \\ Professor ECE \\ JNTU, Hyderabad, India
}

\begin{abstract}
WCDMA is interference limited multiple access technique and mainly used for transmission of high data rate in the 3rd generation cellular and mobile networks. In conventional Call Admission Control (CAC) techniques, when a new call admit in the system to get admission, it checks whether the call is admitted or not on the basis of signal to interference ratio (SIR) and transmission power in the network. If the call is accepted it will create some interference to the ongoing calls during data transmission and reception. This new interference would degrade the performance of ongoing calls; hence the proper Resource Allocation with Call Admission Control in the network is very essential to maintain the communication with guaranteed quality of service (QoS). This paper proposes a novel approach for Resource Allocation with connection admission control (RA-CAC) to maintain present and future target throughput for entire duration of the system which is mainly based on the utility function. The simulation result shows that the proposed Throughput Based Resource Allocation Scheme with connection admission control algorithm can significantly increase packet delivery ratio with less delay and higher throughput.
\end{abstract}

\section{Keywords}

RAN; WCDMA; RACAC; QoS

\section{INTRODUCTION}

WCDMA is a spread-spectrum modulation technique; which uses channels with bandwidth much greater than that of the data to be transferred [1]. Instead of each connection being granted a dedicated frequency band just wide enough to accommodate its envisaged maximum data rate, W-CDMA channels share a much larger band.

The modulation technique encodes each channel in such a way that a decoder, knowing the code, can pick out the wanted signal from other signals using the same band, which simply appear as so much noise.

The wide-band CDMA (W-CDMA) technology is the major air interface for $3 \mathrm{G}$ wireless systems. It provides a transmission rate of $144 \mathrm{Kbps}$ to $2 \mathrm{Mbps}$ and enables multimedia services like the broadband wired networks. WCDMA can support services with higher rate when it is measured up with the narrow-band CDMA. It is adaptable to distribute multimedia traffic. A new medium access control protocol (MAC) is required to manage packet access efficiently in wideband CDMA wireless networks in order to make use of such instruments Efficient Call Admission Control (CAC) and Medium Access Control (MAC) protocols are necessary for the QoS provisioning in WCDMA environment [2].
The rest of the paper is organized as follows: section II gives the details about the literature review. The complete detail about proposed algorithm in section III. Next, section IV gives the simulation result for the proposed approach, finally conclusions are provided in section $\mathrm{V}$.

\section{LITERATURE REVIEW}

Jraifi Abdelouahed [3] has presented the major problem that contributes in limiting the performance of cellular networks. Also an analytical method for studying the co-channel interference in WCDMA system has been developed. A micro zoning architecture to compute the $(\mathrm{S} / \mathrm{I})$ ratio is adopted. This analytical result is used to predict the number of users per micro zones. This result is of crucial importance because it contributes positively in improving the quality of services. However the number users should be controlled for better QoS performance.

N. Mohan and T. Ravichandran [4] ave proposed to design a new CAC algorithm with power control for multiple services like voice, video and data for multiclass users. It determines the optimum set of admissible users with optimum transmitting power level, so as to minimize the interference level and call rejection rate. In addition to this, an adaptive scheduling scheme to allocate optimum rate for each traffic queue is proposed to minimize the scheduling delay. The proposed algorithms achieve reduced call blocking probability, optimum rate with reduced delay. However there is a delay in the scheme.

Salman A. AlQahtani et al [5] have proposed and analyzed an efficient uplink-scheduling scheme in case of RAN sharing method. This new scheme is called as Multioperators Code Division Generalized Processor sharing scheme (M-CDGPS). It employs both adaptive rate allocation to maximize the resource utilization and GPS techniques to provide fair services for each operator. The performance analysis of this scheme is derived using the GPS performance model. Also, it is compared with static rate M-CDGPS scheme. Results show that the proposed adaptive rate MCDGPS scheduling scheme improves both system throughput and average delays. However the theoretical delay bound increases as the token bucket size increases.

Tajje-eddine Rachidi et al [6] have presented QaPC and QaHO mechanisms, which are based on the class of service, the bit rate, and the Service Degradation Descriptor (SDD) as enabling QoS parameters. They have used bit rate, service class and Service Degradation Descriptor for enabling QoS parameters. The proposed QoS aware mechanism which significantly improves QoS contract upholding for premium mobile users, as well as increases resource utilization, while improving SHO acceptance. However there is overload in the system. 
Young-Long Chen et al [7] have proposed a novel approach which combines the CAC and power control mechanisms and operates in a centralized control manner. The essence of the proposed centralized call admission control (CCAC) scheme is to combine the two mechanisms and to treat the call admission decision as an eigen-decomposition problem. In order to reduce the computational complexity of the eigendecomposition problem, the paper proposes an additional scheme, which uses a norm operation rather than direct computation. The proposed scheme, even with the norm approximation, outperforms conventional call admission methods in terms of both its blocking rate and its outage rate. Consequently, the actual SIR of each link in a neighboring base station may not be guaranteed, with the result that outage may occur.

Peifang Zhang and Scott Jordan [8] have constructed a framework for connection access control and rate scheduling. They present a decomposition of the problem into connection access control and rate scheduling layers. Through numerical analysis, they show how wireless channel time diversity and multi-user diversity is exploited to construct a rate scheduling algorithm that is superior to proportional fairness. However the decrease in power makes it more difficult to achieve the targets of all users.

\section{SYSTEM MODEL}

In the proposed framework, new sessions present its utility function to the network to express its satisfaction with the throughput achieved over their connection lifetime. The utility functions are selected by network based on type of application to balance the short-term and long-term goals. The network decides whether to admit or block the new session based on the utility function. If the session is admitted, the network will set a target throughput to be achieved during the lifetime of the session. During each session, the network adjusts the instantaneous rates of each connection based on their target throughput. The main goal is to maximize the long term average utility per unit time by balancing the number of connections admitted, the power dedicated to maximizing achieved throughput, and the power dedicated to ensuring that target throughputs are achieved[9].

If the network admits too many connections, then the utility will fall when the targets are not attained. If the network concentrates more on connections with relatively good channels, then the aggregate throughput will be improved while decreasing the utility. This problem is solved by -

Resource Allocation with Connection Admission Control (RACAC) layer.

In RA-CAC layer, the network decides whether to admit the new sessions or not and according to that it will set the target throughput for those admitted sessions. Off-line decisions based on the past, present and future channel conditions, arrivals and departures. Hence, the online decision is used that considers only the past and present information. However, RA-CAC depends on some future functions. In order to eliminate this dependence by assuming that the RACAC layer will maintain the same target future throughput for the all active user throughout its lifetime.

\section{Algorithm-}

1. accept a new call request

2. Calculate the priority values-

2.1. if ga, $\mathrm{i}<1$ calculate $\mathrm{T} j \&$ fi.
3. allocate the resources with highest priority values

3.1. if still, ga, $i<1$

3.1.1. assign a rate increment $\Delta \mathrm{i}(\mathrm{fi} \leftarrow \mathrm{fi}+\Delta \mathrm{i})$ such that

ga, $\mathrm{i}=1$, admit the request where $\mathrm{fi}=\varphi \mathrm{R}$

else if

$3.2 \mathrm{ga}, \mathrm{i}>1$ then

3.2.1 assign a rate increment such that -

either $\Delta \mathrm{i}=\Delta^{\prime}$ ( a fixed step) or $\mathrm{fi}=\varphi \mathrm{R}$.

end

4. up date the predicted achievement ratio ga,i .

5. network starts to wait for new arrival

5.1. if $\mathrm{Tj}>0$, then

5.1.1. arrival is admitted \& goes to step 1

else if

5.2. if $\mathrm{Tj}<0$, then

5.2.1. arrival is blocked

else

5.3. go to step $2 \& 3$.

6. go to step $1 \& 4$.

\section{SIMULATION RESULTS}

In this section, proposed Throughput Based Resource Allocation with Connection Admission Control (RACAC) for Real-time Traffic in WCDMA cellular networks compare with Centralized Call Admission Control for cellular and mobile networks. The simulation tool used is NS-, which is a general purpose simulation tool that provides discrete event simulation of user defined networks. In the simulation, mobile nodes move in a 600 meter x 600 meter rectangular region for 50 seconds simulation time. Simulation results shows comparison between proposed RACAC scheme against the centralized call admission control (CCAC) scheme.

\subsection{Based on Number of Requests}

In our first experiment, we vary the number of requests from 1 to 6 among 36 Cells.

\section{No. of Users Vs Packet Delivery Ratio}

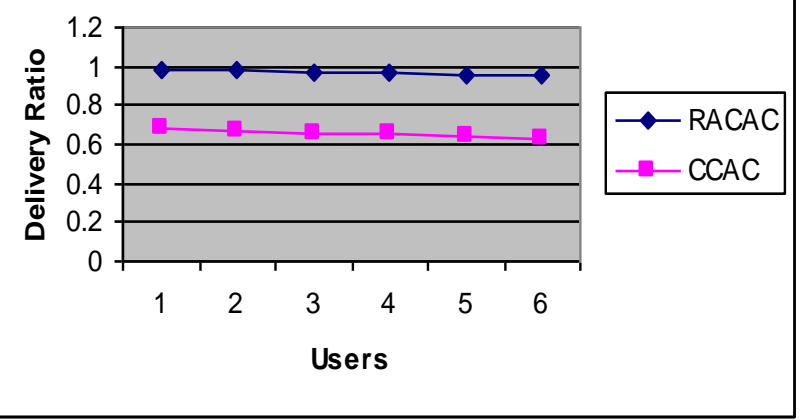

Figure: 1 - Users Vs Delivery Ratio 


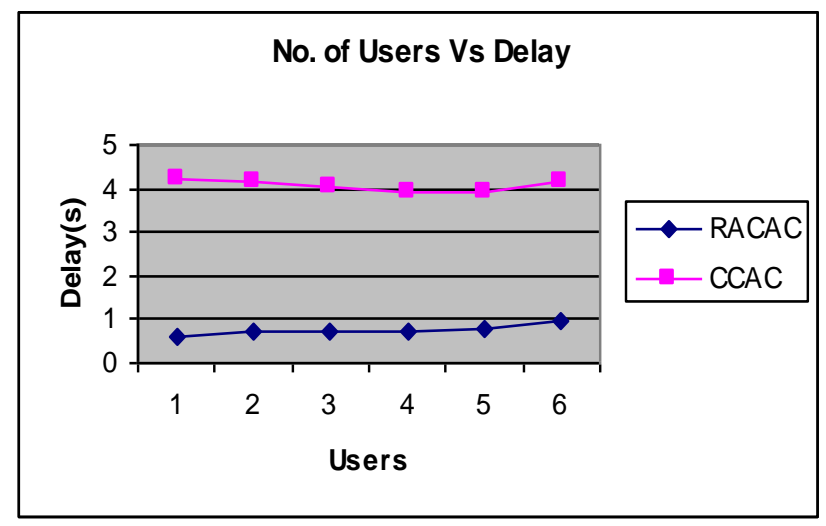

Figure: 2 - Users Vs Delay

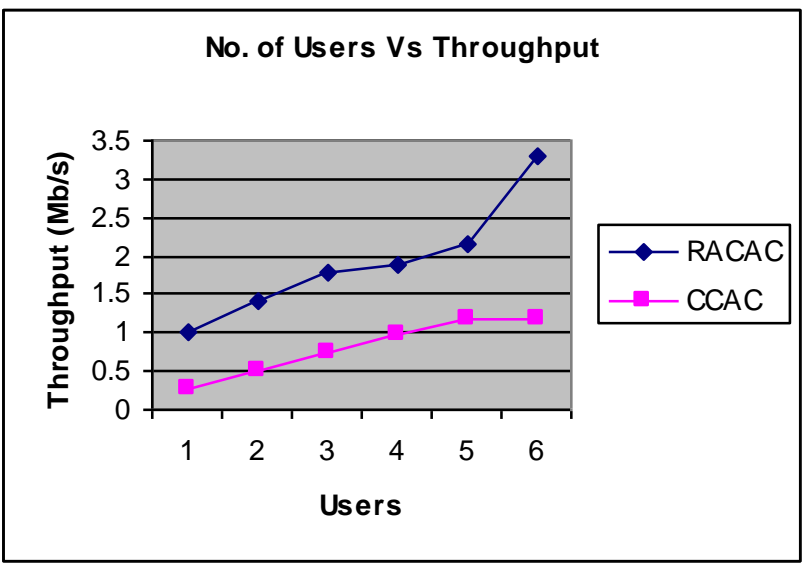

Figure: 3 - Users Vs Throughput

Figure 1 gives the delivery ratio calculated in both the schemes. From the figure, it is clear that our RACAC scheme have $34 \%$ more delivery ratio, when compared with the CCAC scheme. From Figure 2 we can observe that our proposed RACAC has $80 \%$ less delay, when compared with the CCAC scheme. Figure 3 shows the average throughput measured. From the figure, it is apparent that our RACAC scheme attains $74 \%$ more throughputs when compared to the CCAC scheme for cellular and mobile networks.

\section{CONCLUSION}

To go on the interference limitation and enhancing the throughput in WCDMA Networks this paper started with suitable introduction describing with the all factors of QoS issues. Proposed Resource Allocation with Connection Admission Control (RACAC) is done with the RA-CAC layer which improves the packet delivery ratio with less delay and higher throughput as compare than existing Centralized Call Admission Control scheme. The QoS of the real-time multimedia data is improved in an efficient way.
On the whole the performance of the system is achieved with very high throughput and the utilization is done in an useful way. Future works include research on scheduling to reduce congestion and improve QoS in WCDMA Network.

\section{REFERENCES}

[1] N. Mohan \& T. Ravichandran, "An Efficient Multiclass Call Admission Control and Adaptive Scheduling for WCDMA Wireless Network", European Journal of Scientific Research ISSN 1450-216X Vol.33 No.4 (2009).

[2] Omar Younis Alani \& Mohamed Aziz, "Dynamic WCDMA System Proposal for QoS in Multimedia Networks", Computers and Communications (ISCC), 2010 IEEE Symposium on. IEEE, 2010.

[3] Jraifi Abdelouahed, "A Theoretical Prediction of Users in WCDMA Interference", Journal of Theoretical and Applied Information Technology 31st January 2012. Vol. 35 No.2.

[4] N. Mohan and T. Ravichandran, "An Efficient Multiclass Call Admission Control and Adaptive Scheduling For WCDMA Wireless Network", European Journal of Scientific Research 33.4 (2009).

[5] Salman A. AlQahtani, Ashraf S. Mahmoud and Asrar U. Sheikh, "Performance Analysis of Adaptive Rate Scheduling Scheme for 3G WCDMA Wireless Networks with Multi-Operators", Communications, 2007. ICC'07. IEEE International Conference on. IEEE, 2007.

[6] Tajje-eddine Rachidi, Amal Yasmine Elbatji, Mehdi Sebbane and Hicham Bouzekri, "QoS-Aware Power Control and Handoff Prioritization In 3g WCDMA Networks", Wireless Communications and Networking Conference, 2004. WCNC. 2004 IEEE. Vol. 2. IEEE, 2004.

[7] Young-Long Chen, Jyu-Wei Wang, Jyh-Liang Chen and Jyh-Horng Wen, "Performance of Call Admission Control with Power Control in Multimedia Cellular Systems", International Journal of Applied Science and Engineering 5.1 (2007)

[8] Peifang Zhang and Scott Jordan, "Cross Layer Dynamic Resource Allocation with Targeted Throughput for WCDMA Data", IEEE Transactions on Wireless Communications, Vol. 7, No. 12, December 2008.

[9] Leonardo Badia, Adriana Telesca and Michele Zorzi, "On the Network Utility Optimal Allocation of Radio Resources in WCDMA Systems", IEEE Communications Letters, Vol. 10, No. 5, May 2006. 